\title{
Umsetzung nationaler Massnahmenplan läuft an
}

\section{Gert Printzen}

Dr. med., Mitglied des Zentralvorstandes der FMH, Departementsverantwortlicher eHealth - Medizinische Informatik und Dokumentation / Heilmittel

\author{
Das Bundesamt für Gesundheit hat die Umsetzung des Nationalen Konzepts \\ Seltene Krankheiten in vier Teilprojekte gebündelt. Die Umsetzung der geplanten \\ Verbesserungen ist eine grosse Herausforderung, einzelne Fragen sind jedoch noch \\ nicht abschliessend geklärt. Das Postulat von Nationalrätin Ruth Humbel sollte \\ deshalb noch nicht «abgeschrieben» werden.
}

Bund und Kantone haben bereits am 19. Februar 2015 im Rahmen des «Dialogs Nationale Gesundheitspolitik» über die ersten Schritte bei der Umsetzung des Nationalen Konzepts Seltene Krankheiten diskutiert. Öffentlich orientierte Oliver Peters, Vizedirektor des Bundesamts für Gesundheit (BAG), am diesjährigen Tag der Seltenen Krankheiten Ende Februar in Fribourg erstmals über die seitens des BAG geplanten weiteren Schritte.

\section{Umsetzung des nationalen Konzepts in vier Teilprojekten}

Die im Nationalen Konzept Seltene Krankheiten vorgeschlagenen 19 Massnahmen sollen in vier Teilprojekte gebündelt und weiter bearbeitet werden.

Im Teilprojekt 1 «Diagnose» steht der Aufbau der natio-

\section{Die FMH als zentraler Akteur ist aus unserer} Sicht für die Mitarbeit in allen vier Teilprojekten vorzusehen.

nalen Referenzzentren für seltene Krankheiten sowie der Prozess für die Auswahl und Evaluation dieser Referenzzentren im Mittelpunkt. Erarbeitet werden sollen hier zudem der Aufbau von nationalen Registern, die Harmonisierung der Daten und Codierungen sowie ein besserer Informations- und Wissenstransfer zwischen den Spezialisten.

Teilprojekt 2 «Zugang» befasst sich mit der Kostenübernahme bei Patienten, der Überprüfung der Geburtsgebrechenliste (GGL) sowie der Spezialitätenliste für Medikamente (SL). Diese Arbeitsgruppe existiert bereits, erste Resultate sollen bald vorliegen.

Teilprojekt 3 «Unterstützung und Angehörige» bein- haltet Massnahmen zur Verbesserung des Informationstransfers zwischen den einzelnen beteiligten Akteuren sowie die bessere Koordination zwischen Kantonen und Spitälern, den helfenden Angehörigen und einem verbesserten Einbezug der Patientenorganisationen.

Teilprojekt 4 «Forschungszugang» behandelt insbesondere die Förderung und Stärkung der universitären Ausbildung von Ärztinnen und Ärzten im Bereich der seltenen Krankheiten sowie die Förderung der diesbezüglichen klinischen Forschung in der Schweiz.

Für die Erarbeitung der vier Teilprojekte sollen jeweils von den Themen direkt betroffene Akteure einbezogen werden. Diese Expertengruppen bearbeiten die einzelnen Teilprojekte unter der Federführung des BAG parallel. Zur Ausrichtung und Justierung der Inhalte sind jährliche Workshops geplant. Die FMH als zentraler Akteur ist aus unserer Sicht für die Mitarbeit in allen vier Teilprojekten vorzusehen, die zwischen 2015 und 2017 umgesetzt werden.

\section{Einzelne Fragen bleiben noch offen}

Von der Einreichung des Postulats von Ruth Humbel im Jahre 2010 bis zum nun vorliegenden Umsetzungsvorschlag lag ein langer Weg. Nach einiger Vorbereitungszeit führte das BAG von Herbst 2013 bis Frühling 2014 vier Workshops mit allen betroffenen Organisationen durch. Die vom BAG präsentierten Ziele, Handlungsfelder und Prioritäten entsprachen in den wesentlichen Teilen denjenigen des ersten Entwurfs für einen nationalen Massnahmenplan der IG Seltene Krankheiten (IGSK) aus dem Jahre 2012. Im Herbst 2014 präsentierte das BAG dann das Nationale Konzept Seltene Krankheiten, nun liegen die Umsetzungsprojekte 
vor. Etliche wichtige Elemente und Forderungen sind darin enthalten. So ist die Bündelung von Ressourcen und Kompetenzen im Bereich seltene Krankheiten in nationalen Referenzzentren weitgehend unbestritten. Ebenso der verbesserte Zugang zu Diagnosen und die Stärkung des Informations- und Wissenstransfers sowie die Stärkung der Unterstützung der Angehörigen und Patientenorganisationen und die internationale Zusammenarbeit. Diverse Punkte aus dem Entwurf eines nationalen Massnahmenplans der IGSK blieben jedoch unberücksichtigt.

Die vier Teilprojekte mit insgesamt 19 Massnahmen des Bundes im Rahmen des Nationalen Konzepts Seltene Krankheiten sind zu unterstützen. Bei weiteren zentralen Punkten wird es eine grosse Herausforderung bleiben, gemeinsam konkrete Lösungsvorschläge zu finden, so z.B. bei der Sicherstellung des rechtsgleichen Zugangs zu integralen Therapien und deren Vergütung, bei der einheitlichen Regelung des Übertritts von der IV in die obligatorische Krankenversicherung, bei der Mitsprache der Fachärzte bei Vergütungsentscheiden sowie bei der Förderung der Grundlagenforschung neben der klinischen und der translationalen Forschung.

Die vorgeschlagenen Massnahmen sind nun zu präzisieren und pragmatisch und flexibel umzusetzen, um zu einer nachhaltigen Verbesserung der Situation betroffener Menschen beizutragen. Oft sind dabei Details entscheidend. Wichtig ist dabei insbesondere die klare Definition von Kompetenzen und Zuständigkeiten bei den einzelnen Massnahmen sowie deren Finanzierung. Der bisherige Prozess zeigt, dass der Erarbeitungs- und Implementierungsprozess breit abgestützt mit allen betroffenen Stakeholdern erfolgen sollte, um bestmögliche, tragfähige Lösungen zu ermöglichen. Der Prozess zur Umsetzung des nationalen Massnahmenplans in Deutschland mag hier als gutes Beispiel dienen. Das Bundesministerium für Gesundheit, das Bundesministerium für Bildung und Forschung sowie die Interessenvertretungen der Betroffenen arbeiten dort partnerschaftlich und auf gleicher Augenhöhe im Nationalen Aktionsbündnis für
Menschen mit Seltenen Erkrankungen NAMSE zusammen. Es ist zu wünschen, dass auch in der Schweiz bei der weiteren Umsetzung auf eine enge Zusammenarbeit und Kooperation mit allen betroffenen Stakeholdern gesetzt wird und die Anliegen aller betroffenen Akteure ernst genommen werden.

\section{Postulat von Ruth Humbel noch nicht abschreiben}

In ihrem Postulat 10.4055 n vom 27. November 2010 fordert Nationalrätin Ruth Humbel den Bundesrat auf, einen nationalen Aktionsplan zu erstellen. Einzelne Forderungen des Postulats sind auf gutem Weg, insbesondere die nationalen Referenzzentren für seltene Krankheiten. Andere zentrale Forderungen werden mit den vier Teilprojekten noch nicht vollständig abgedeckt, so die Gewährleistung des rechtsgleichen $\mathrm{Zu}$ gangs zu Diagnostik und wirksamen Therapien, sowie die Priorisierung seltener Krankheiten in der Grundlagenforschung und der klinischen Forschung. Es ist vorhersehbar, dass bei der Umsetzung mit vielfältigen Vollzugsproblemen gerechnet werden muss. Diese Herausforderungen sind zu meistern, wenn alle Akteure gewillt sind, partnerschaftlich zusammenzuarbeiten, damit nachhaltige Verbesserungen für alle Betroffenen, insbesondere der rechtsgleiche Zugang zu wirksamen, evidenzbasierten Therapien und Arznei-

Wichtig ist insbesondere die klare Definition von Kompetenzen und Zuständigkeiten bei den einzelnen Massnahmen sowie deren Finanzierung.

mitteln, möglich werden. Der Handlungsbedarf und der Leidensdruck bei den Betroffenen bleiben hoch. Das Postulat Humbel soll deshalb noch nicht abgeschrieben werden, bis die Erfüllung der darin formulierten Forderungen gesichert ist. 